\title{
Solution processible organic transistors and circuits based on a $\mathrm{C}_{70}$ methanofullerene
}

\author{
Thomas D. Anthopoulos, ${ }^{\text {a) }}$ Dago M. de Leeuw, and Eugenio Cantatore \\ Philips Research Laboratories, Professor Holstlaan 4 (WAG 11), 5656 AA Eindhoven, \\ The Netherlands \\ Patrick van 't Hof, Jan Alma, and Jan C. Hummelen

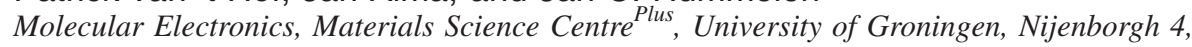 \\ 9747 AG Groningen, The Netherlands
}

(Received 16 March 2005; accepted 19 July 2005; published online 8 September 2005)

\begin{abstract}
We demonstrate ambipolar charge transport in organic field-effect transistors based on the solution processible methanofullerene $[6,6]$-phenyl- $\mathrm{C}_{71}$-butyric acid methyl ester ([70]PCBM). The electron mobility measured in the linear and saturated regimes is approximately equal and of the order of $1 \times 10^{-3} \mathrm{~cm}^{2} / \mathrm{V}$ s. In the linear regime, mobility is thermally activated with a strong gate bias dependence. The $p$-channel hole mobility is lower with a maximum value of $2 \times 10^{-5} \mathrm{~cm}^{2} / \mathrm{V}$ s. The $n$ - and $p$-channel operations are characterized by a low $(\sim|3| \mathrm{V})$ and a high $(\sim|50| \mathrm{V})$ switch-on voltage, respectively, with the on-off current ratio on the order of $10^{4}$ for both channels. Although the present findings are qualitatively similar to those reported previously for $\mathrm{C}_{60}$-methanofullerene-based transistors, [70]PCBM devices are more stable and easier to reproduce. By making use of this property we are able to demonstrate functional unipolar and complementarylike logic circuits with promising operating characteristics. () 2005 American Institute of Physics. [DOI: 10.1063/1.2034083]
\end{abstract}

\section{INTRODUCTION}

In recent years organic field-effect transistors (OFETs) are receiving much attention as possible alternatives to inorganic devices in a range of low-end electronic applications. ${ }^{1-5}$ Over the past ten years, progress in OFETs is driven by the improvement in the charge-carrier mobility ${ }^{4}$ and by scaling of lateral dimensions. ${ }^{6}$ In particular, the discovery and development of high mobility organic semiconductors has been proven to be invaluable for fabrication of organic circuits suitable for low-end device applications. ${ }^{5,7-11}$ Most of these organic semiconductors are $p$-channel materials $\mathrm{s}^{1-7,9,10}$ with maximum mobility of $15 \mathrm{~cm}^{2} / \mathrm{V} \mathrm{s}$ (Ref. 12) and only a small fraction is found to function as $n$-channel semiconductors. ${ }^{4,8,11,13,14}$ Apart from their use in unipolar circuits, $n$-channel transistors are important because when combined with their $p$-channel counterparts they can form the basis of complementary integrated circuits similar to those found in contemporary silicon solid-state electronics.

Fullerenes and, in particular, $\mathrm{C}_{60}, \mathrm{C}_{70}, \mathrm{C}_{59} \mathrm{~N}, \mathrm{Dy}-\mathrm{C}_{82}$, $\mathrm{La}_{2}-\mathrm{C}_{80}$, and $\mathrm{C}_{84}$ (Refs. 14-19) are among the known $n$-type organic semiconductors with $\mathrm{C}_{60}$ exhibiting the highest mobility of $\sim 0.5 \mathrm{~cm}^{2} / \mathrm{V} \mathrm{s}^{20}$ Although fullerenes are known to be very sensitive to oxygen, ${ }^{14-19}$ recent work has demonstrated that $\mathrm{C}_{60}$ OFETs can be efficiently passivated. ${ }^{21}$ This makes fullerenes strong candidates for use in future organic-based electronic circuits. ${ }^{11}$

Recently, fabrication of organic transistors based on the solution processible methanofullerene [6,6]-phenyl-

\footnotetext{
${ }^{a)}$ Author to whom correspondence should be addressed; electronic mail: thomas.anthopoulos@philips.com
}

$\mathrm{C}_{61}$-butyric acid methyl ester ([60]PCBM) has been reported. ${ }^{22-24}$ Methanofullerenes possess an advantage over pristine fullerenes. Although their electronic properties are similar to those of unsubstituted fullerenes, i.e., they are characterized by relatively high electron mobility [1 $\times 10^{-2}-2 \times 10^{-1} \mathrm{~cm}^{2} / \mathrm{V} \mathrm{s}$ (Refs. 22-24) compared to 5 $\times 10^{-1} \mathrm{~cm}^{2} / \mathrm{V}$ s for pristine $\mathrm{C}_{60}$ (Ref. 20)], their intermolecular interactions, as well as the processing properties (solubility, etc.), can be tailored accordingly by the type of substitution on the periphery of the carbon cage. Additionally the [60]PCBM derivative was found to exhibit ambipolar charge transport characteristics, i.e., selective or simultaneous transport of electrons and/or holes. ${ }^{23}$ As a result the operation of a [60]PCBM-based complementarylike inverter, the building block of logic circuits, was demonstrated. ${ }^{25}$ Despite these promising preliminary results, however, no higher methanofullerene analogues have yet being studied as potential electroactive materials in organic transistors.

Here, we extend our previous studies and we report on field-effect transistors based on the solution processible methanofullerene [6,6]-phenyl- $\mathrm{C}_{71}$-butyric acid methyl ester ([70]PCBM). The latter is a higher analogue of [60]PCBM and its synthesis was performed in an analogous procedure. $^{26,27}$ In particular, we show that bottom contact [70]PCBM transistors exhibit $n$ - and $p$-channel characteristics, qualitatively similar to those reported for [60]PCBM devices. ${ }^{23}$ We note, however, that preparation of [70]PCBM transistors is much easier than [60]PCBM-based devices and exhibit high reproducibility. Due to this easy to fabricate nature of [70]PCBM transistors we are able to demonstrate unipolar ring oscillators as well as complementarylike voltage inverters. The present findings are interesting not only 


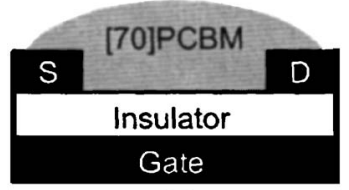

(a)

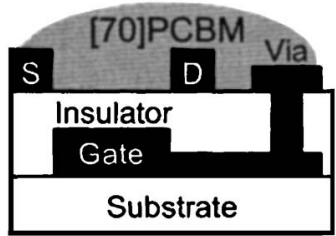

(b)

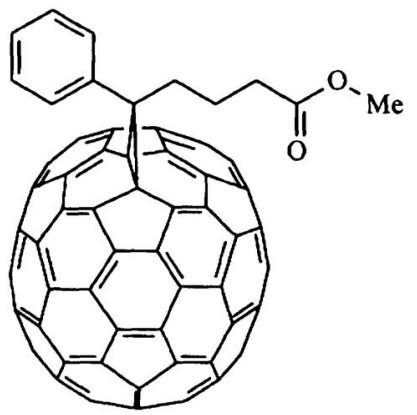

(c)

FIG. 1. (a) Device structure of discrete OFET with symmetric gold electrodes and $\mathrm{SiO}_{2}$ as the gate dielectric. (b) OFET structure employed for the fabrication of the seven-stage ring oscillator. (c) Molecular structure of [70]PCBM.

for their potential in technological applications but also for the fundamental understanding of methanofullerenes.

\section{EXPERIMENTAL DETAILS}

Discrete OFETs [Fig. 1(a)] were made using heavily doped $p$-type $\mathrm{Si}$ wafers as the back gate electrode with a 200-nm thermally oxidized $\mathrm{SiO}_{2}$ layer as the gate dielectric. Using conventional photolithography, gold source and drain electrodes were defined with channel length and width in the range of $1.5-40 \mu \mathrm{m}$ and $1-20 \mathrm{~mm}$, respectively. A $10-\mathrm{nm}$ layer of titanium was used as an adhesion layer for the gold on the $\mathrm{SiO}_{2}$. The $\mathrm{SiO}_{2}$ layer was treated with the primer hexamethyldisilazane prior to semiconductor deposition. The detailed fabrication process of integrated circuits is described elsewhere. ${ }^{5}$ In brief, gate electrodes and first-level interconnect lines were made by patterning gold using standard photolithography techniques. The gate dielectric was a 300-nm-thick photoimageable polymer, which was spin cast and subsequently exposed to ultraviolet light to define contact holes. Source and drain electrodes and second-level interconnects were defined in the second gold layer [Fig. 1(b)]. Semiconductor films were drop cast from a $10-\mathrm{mg} / \mathrm{ml}$ solution of [70]PCBM in chlorobenzene with the substrate maintained at $60{ }^{\circ} \mathrm{C}$. All devices were annealed in vacuum $\left(10^{-5} \mathrm{mbar}\right)$ at $115^{\circ} \mathrm{C}$ for several hours prior to characterization. Electrical measurements were performed using an HP 4156B semiconductor parameter analyzer with the sample maintained at $40^{\circ} \mathrm{C}$ in vacuum $\left(10^{-5} \mathrm{mbar}\right)$.
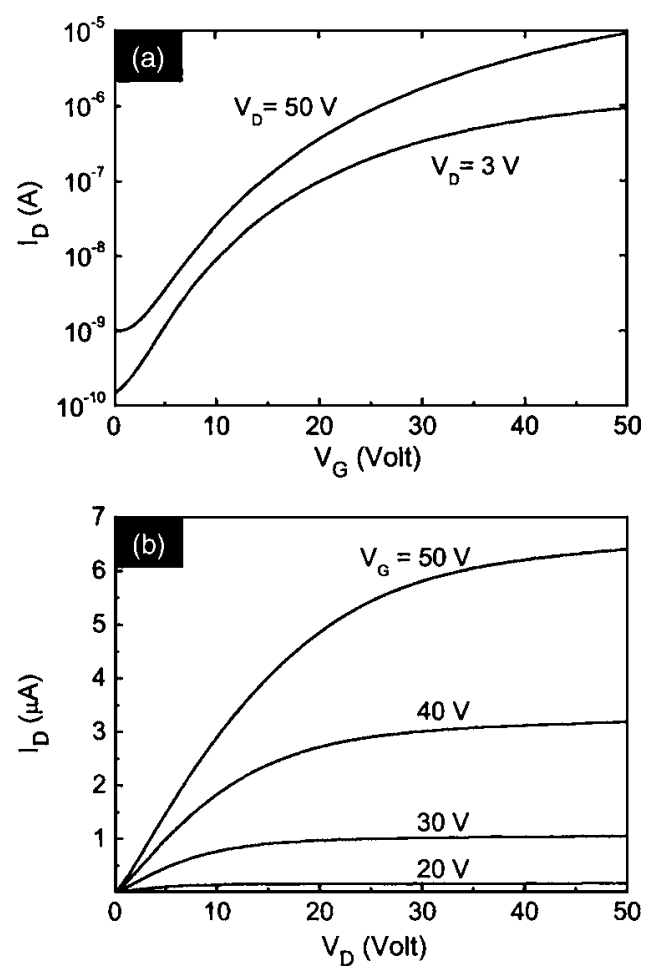

FIG. 2. (a) Transfer characteristics of a discrete [70]PCBM transistor with $L=7.5 \mu \mathrm{m}$ and $W=10 \mathrm{~mm}$ in the $n$-channel regime. (b) Output characteristics of the same device.

\section{RESULTS AND DISCUSSION}

\section{A. Charge transport in [70]PCBM transistors}

Figure 1(a) shows the schematic cross section of the discrete OFETs employed. The molecular structure of [70]PCBM is shown in Fig. 1(c) and represents the main isomer among a mixture of isomeric methanofullerenes present in our material. ${ }^{27}$ Although [70]PCBM resembles [60]PCBM, their electronic properties are expected to be different. For instance, in the case of unsubstituted $\mathrm{C}_{70}$, the molecule is slightly larger in diameter than $\mathrm{C}_{60}$ leading to smaller electron-electron repulsion energy. ${ }^{28}$ Moreover, pristine $\mathrm{C}_{70}$ is known to have a slightly lower ionization potential than $\mathrm{C}_{60}$ by approximately $100 \mathrm{mV} .^{29}$ For organic electronic devices and, in particular, transistors, this relatively small difference may have a large impact on the carrier injection efficiency.

Figure 2(a) shows the $n$-channel transfer characteristics for a $[70] \mathrm{PCBM}$ transistor [Fig. 1(a)] with channel length $L$ and width $W$ of $7.5 \mu \mathrm{m}$ and $10 \mathrm{~mm}$, respectively. The device exhibits a low switch-on voltage $\left(V_{\mathrm{SO}}\right)$ even after a relatively short annealing period of $2 \mathrm{~h}$ at $115^{\circ} \mathrm{C}$ in vacuum without any significant hysteresis. We note that for [60]PCBM transistors heat treatment is a much lengthier process requiring annealing times in excess of $20 \mathrm{~h}$. This difficulty in reproducing reliable [60]PCBM transistor is a limiting factor for their use in practical electronic applications.

Upon exposure of the [70]PCBM transistor to air degradation in the device performance is observed. In particular, the drain current, at $V_{D}=V_{G}=50 \mathrm{~V}$, is reduced by more than two orders of magnitude within the first 5 min of exposure while the threshold voltage increases to $>20 \mathrm{~V}$. Degrada- 
tion, however, for [70]PCBM is less rapid than in the case of [60]PCBM (Ref. 23) where the field effect was found to completely vanish within the first few minutes of device exposure to atmospheric air. The charge-carrier mobility was measured in the linear and saturation regimes using the local approximation method. ${ }^{30}$ In the linear regime $\left(V_{D} \rightarrow 0\right)$ the carrier mobility is calculated from

$$
\mu=\frac{L}{C_{i} W V_{D}} \cdot \frac{\partial I}{\partial V_{G}}
$$

where $C_{i}$ is the geometric capacitance of the dielectric. Using Eq. (1) together with the data of Fig. 2(a), for $V_{D}=3 \mathrm{~V}$, a maximum mobility of $1 \times 10^{-3} \mathrm{~cm}^{2} / \mathrm{V} \mathrm{s}$ is calculated. In the saturation regime the mobility was calculated from

$$
\mu=\frac{L}{C_{i} W} \cdot \frac{\partial^{2} I}{\partial V_{G}^{2}}
$$

Equation (2) together with the data of Fig. 2(a), for $V_{D}$ $=50 \mathrm{~V}$, yields a maximum saturated field-effect mobility of $2 \times 10^{-3} \mathrm{~cm}^{2} / \mathrm{V} \mathrm{s}$. In spite this relatively low value we establish that $[70]$ PCBM transistors can be fabricated very reliably with a small parameter spread (mobility, threshold voltage, etc.) This has very important consequences on circuit design.

The electron mobility value in [70]PCBM is typically lower than the value reported for transistors based on [60]PCBM by one order of magnitude. ${ }^{23}$ A plausible explanation could be that such difference is attributed to intrinsic differences in the electronic properties of the two methanofullerenes and particularly the $\mathrm{C}_{60}$ and $\mathrm{C}_{70}$ carbon cages. ${ }^{29}$ For instance, the electronic structure of pristine $\mathrm{C}_{60}$ is known to be isotropic with the lowest unoccupied molecular orbital (LUMO) level delocalized over the whole molecule. ${ }^{31}$ The electronic structure of $\mathrm{C}_{70}$ on the other hand is different with the LUMO having nodes at 30 of the 70 carbon atoms. ${ }^{15}$ Hence, intermolecular interactions (intermolecular overlap of LUMOs in this particular case) will be reduced leading to a less developed dispersion in the conduction band. We stress, however, that quantitative comparison between pristine fullerenes and methanofullerenes should be avoided due to symmetry differences between the two but also due to the presence of a mixture of isomeric methanofullerenes in the case of [70]PCBM. We must also bear in mind that charge transport in spin-coated films of methanofullerenes is possibly dominated by disorder. Therefore, observed differences in the carrier mobility between the various systems may well be attributed to fabrication-related defects rather than the electronic structure of the molecules in question.

The relatively low $n$-channel on-off current ratio of $10^{4}$ (defined as the ratio of $I_{D}$ at maximum $V_{G}$ over $I_{D}$ at $V_{G}$ $\left.=V_{\mathrm{SO}}\right)$ in [70]PCBM transistors [Fig. 2(a)] might be due to differences in the energy gap of $\mathrm{C}_{70}$ (Ref. 29) and/or unintentional doping of [70]PCBM induced either during device preparation or material synthesis. Figure 2(b) shows the typical output voltage characteristics of the [70]PCBM transistor. At $V_{G}>0 \mathrm{~V}$ strong electron-accumulation behavior is observed. In particular, for low $V_{D}$ a linear operating regime is
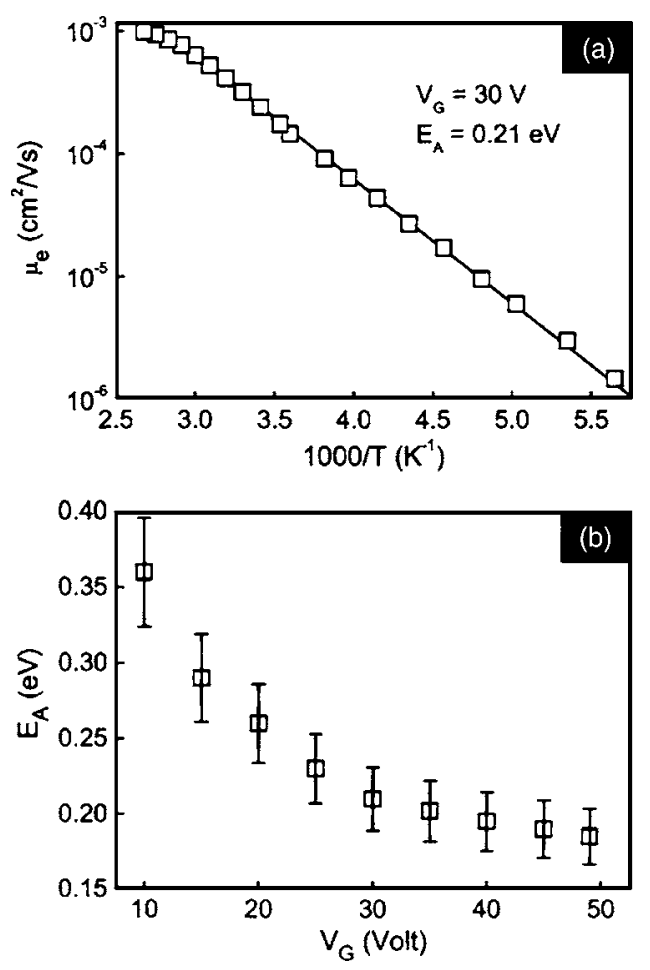

FIG. 3. (a) Arrhenius plot of electron mobility $\left(\mu_{e}\right)$ vs $1000 / T$ at $V_{D}=3 \mathrm{~V}$ and $V_{G}=30 \mathrm{~V}$. The line is Arrhenius fit with activation energy of $0.21 \mathrm{eV}$. (b) Activation energy as a function of gate voltage.

evident followed by a saturation region. A very important feature of these characteristics is that no significant hysteresis is observed.

In order to obtain more insights into the electrontransport process in [70]PCBM transistors, we studied the operating characteristics as a function of temperature in the range of $180-360 \mathrm{~K}$. The linear mobility was measured with $V_{D}=3 \mathrm{~V}$ at gate bias of $30 \mathrm{~V}$ at stabilized temperatures using Eq. (1). These results are shown in Fig. 3(a) where the electron mobility $\left(\mu_{e}\right)$ is plotted as a function of the inverse temperature $(1000 / T)$. The data are consistent with an Arrhenius-type behavior $\left(\mu_{e} \propto \exp \left[-E_{A} / k T\right]\right)$ with activation energy $E_{A}=210 \mathrm{meV}$. The measured value of $E_{A}$ is possibly associated either with the thermally activated hopping transport of charges between localized states and/or to a thermally activated injection barrier at the gold/semiconductor interface. To investigate this we have measured the $E_{A}$ as a function of $V_{G}$. These results are shown in Fig. 3(b). The significance of this plot is that the activation energy decreases with increasing gate voltage reaching a plateau at high $V_{G}$. Although this type of behavior is qualitatively consistent with the predictions of the percolative variable range hopping theory, ${ }^{32}$ fitting of the experimental data in different temperatures and gate bias regimes becomes problematic due to the presence of a contact barrier that is not taken into account. In a try to elucidate the exact mechanism we have calculated the contact resistance $R_{C}$ (source+drain resistance) in our devices using the transistor scaling method. To do so we first calculated the total device resistance $\left(R_{\mathrm{ON}}\right)$, in the linear operating regime $\left(V_{D} \ll V_{G}\right)$, given as $R_{\mathrm{ON}} \equiv \partial I_{D} / \partial V_{D} \quad\left(R_{\mathrm{ON}}\right.$ $=R_{C}+R_{\mathrm{CH}}$ where $R_{\mathrm{CH}}$ is the channel resistance) and plotted it against the channel length. ${ }^{23,33}$ From the intercept of the ex- 

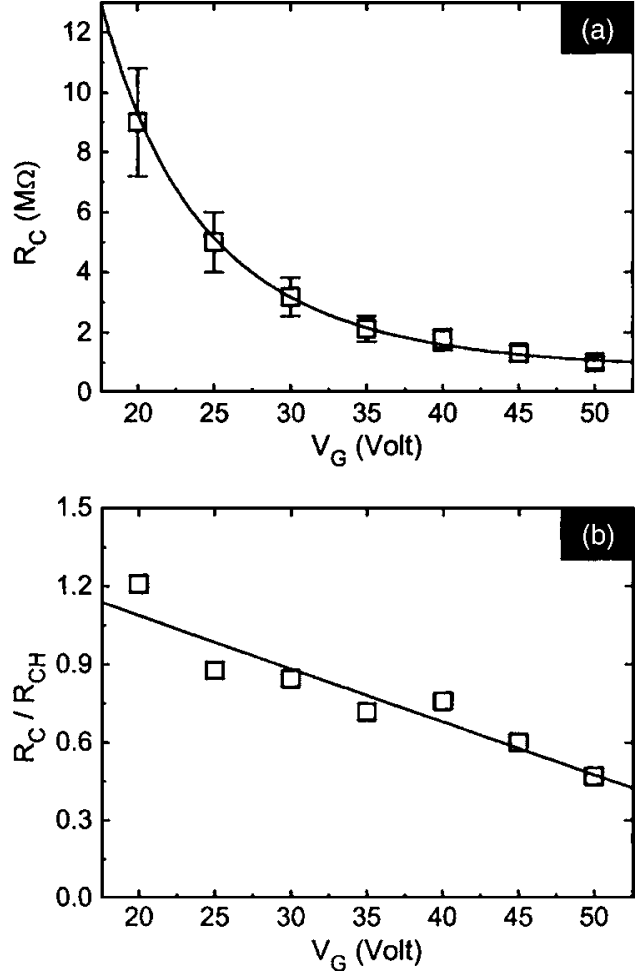

FIG. 4. (a) Contact resistance $R_{C}$ vs gate bias for [70]PCBM-based transistors and (b) ratio of $R_{C}$ over channel resistance $R_{\mathrm{CH}}$ as a function of gate bias. Solid lines are guide to the eye. Scaling behavior was investigated employing a series of [70]PCBM transistors with channel length $L$ in the range of 3-40 $\mu \mathrm{m}$ and constant width $W=10 \mathrm{~mm}$.

trapolated characteristics at $L=0 \mu \mathrm{m}$ with the $y$ axis the value for $R_{C}$ can be obtained at different gate biases, whereas from the slope of the characteristic the value for $R_{\mathrm{CH}}$ per unit channel length is obtained. Figure 4(a) shows the obtained results where the calculated $R_{C}$ is plotted as a function of $V_{G}$. From this figure it is evident that $R_{C}$ reduces as the gate bias increases. Interestingly, $R_{C}$ appears to dominate the device operation for $V_{G}<25 \mathrm{~V}$ [Fig. 4(b)] where $R_{C}$ becomes larger than $R_{\mathrm{CH}}$. Under these conditions we conclude that the description of our devices based solely on the variable-rangehopping $(\mathrm{VRH})$ model, at least for $V_{G}<30 \mathrm{~V}$, is inaccurate and further work is needed in order to elucidate the exact physical mechanism responsible for this effect.

The recent discovery of ambipolar transport in $[60] \mathrm{PCBM}$ transistors $^{23}$ prompted us to investigate the $p$-channel activity in [70]PCBM transistors. Figure 5(a) shows the transfer characteristics for a transistor ( $L$ $=7.5 \mu \mathrm{m}, W=10 \mathrm{~mm}$ ) at negative drain and gate biases. The hysteresis in these curves is found to be very significant and highly dependent upon the experimental conditions, i.e., background pressure and temperature. Nevertheless, typical operating characteristics reveal hole accumulation for $\left|V_{G}\right|$ $>50 \mathrm{~V}$ with the on-off current ratio in the order $10^{4}$. The large current measured for gate bias close to zero is due to electron flow. ${ }^{23,34-36}$ The large value of $V_{\text {SO }}$ is explained conventionally by the presence of a significant density of traps at the insulator/semiconductor interface and the large potential barrier for hole injection. Because of this injection barrier we were unable to accurately determine the hole mobility in the
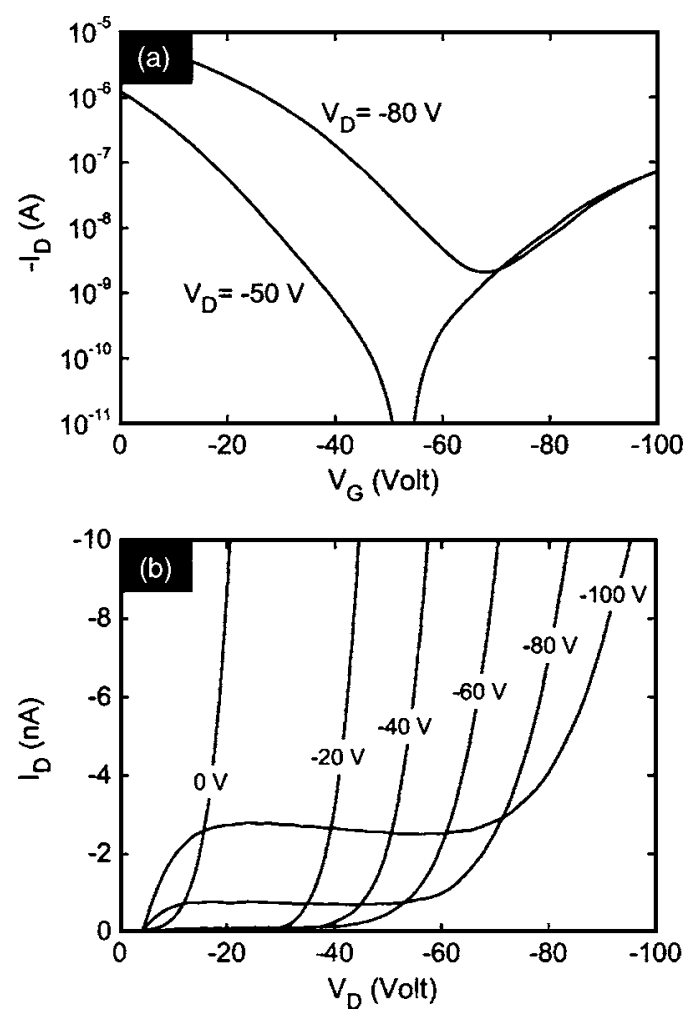

FIG. 5. (a) Transfer characteristics of a discrete [70]PCBM transistor $(L$ $=7.5 \mu \mathrm{m}, W=10 \mathrm{~mm}$ ) operating in the $p$-channel regime at different gate voltages. (b) Output characteristics of the same device.

linear operating regime. Moreover, we find that short channel devices exhibit lower $V_{\mathrm{SO}}$ as compared to longer channel devices. A possible reason for this observation might be the creation of trap states at the grain boundaries of the semiconductor film upon annealing. The saturated field-effect mobility for holes, measured at $V_{D}=-50 \mathrm{~V}$, is $2 \times 10^{-5} \mathrm{~cm}^{2} / \mathrm{V} \mathrm{s}$. This value is lower by more than two orders of magnitude when compared with the hole mobility in [60]PCBM transistors. $^{23}$

Figure 5(b) shows the output voltage characteristics in $p$-channel operation. When the gate voltage $\left|V_{G}\right|>80 \mathrm{~V}$ strong hole accumulation is observed. This behavior originates from holes being injected from the gold into the highest occupied molecular orbital (HOMO) of [70]PCBM and transported across the channel. As $V_{D}$ increases further a pronounced nonlinear increase in the $I_{D}$ is observed without any signs of saturation. This nonlinear increase is due to electron injection from the drain contact. ${ }^{23,34}$ The same is valid for all curves with $\left|V_{G}\right|>0 \mathrm{~V}$ although it is not visible due to the scale of the graph. Finally, for the $V_{G}=0 \mathrm{~V}$ curve the measured current is entirely due to electron flow as depicted from Fig. 5(a).

\section{B. Logic circuits based on [70]PCBM transistors}

We also examined the possibility of using [70]PCBM transistors as the building blocks in integrated circuits. We first fabricated unipolar ( $n$-type) ring oscillators. Because of the switching characteristics of [70]PCBM transistors (i.e., cutoff at $V_{G}=\sim 0 \mathrm{~V}$ ) our design is based on the diodeconnected load logic. ${ }^{37}$ The schematic cross section of the 

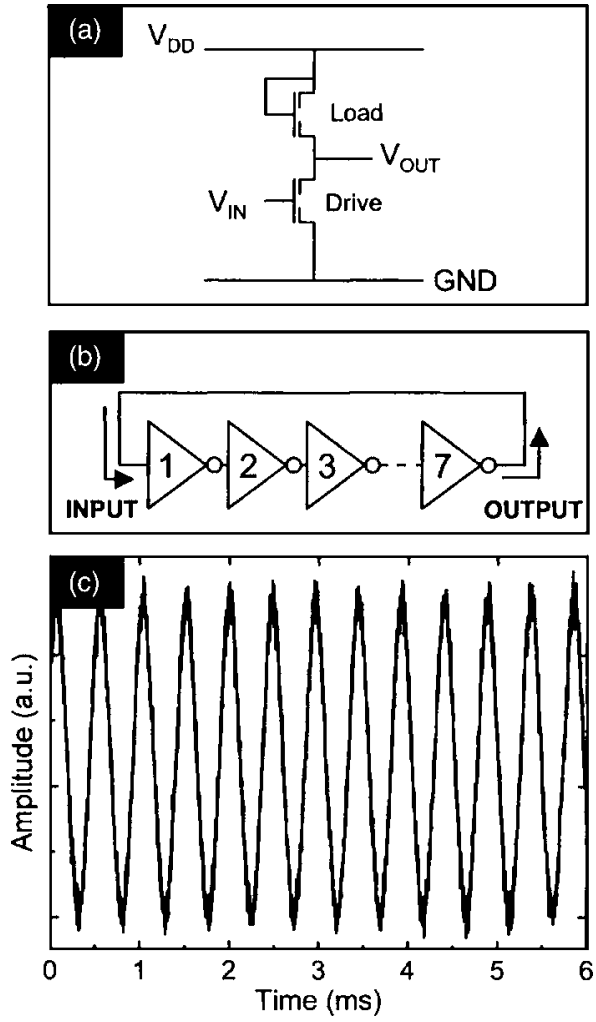

FIG. 6. (a) Schematic circuit of the diode-connected load inverter employed (b) Circuit diagram of the ring oscillator where each triangle represents one inverter. (c) Output signal of a seven-stage [70]PCBM-based ring oscillator. The maximum oscillation frequency measured is $2.1 \mathrm{kHz}$ at a $V_{\mathrm{DD}}=130 \mathrm{~V}$.

individual OFETs employed for the construction of these circuits is shown in Fig. 1(b), while the inverter and ring oscillator circuits are shown in Figs. 6(a) and 6(b), respectively. The output of the last inverter $\left(V_{\text {OUT }}\right)$ is fed back to the input of the first $\left(V_{\mathrm{IN}}\right)$. Hence the circuit oscillates only when an odd number of inverting stages is employed.

In the present work we characterized several sevenstage-ring oscillators [Fig. 6(b)] under different biasing conditions at room temperature. Our faster oscillator circuit is constructed employing a design rule of $1.5 \mu \mathrm{m}$ (Ref. 37) with driver, load, and buffer transistor widths of 2000, 250, and $5000 \mu \mathrm{m}$, respectively. Frequency measurements were performed by measuring the current flowing through a buffer transistor whose gate was driven by the oscillator output using an oscilloscope with $1 \mathrm{M} \Omega$ input impedance. The maximum oscillation frequency $\left(f_{\text {OSC }}\right)$ measured was $2.1 \mathrm{kHz}$ [Fig. 6(c)] at $V_{\mathrm{DD}}=+130 \mathrm{~V}$. The signal propagation delay per stage $(\tau)$ was then calculated using the relation $\tau$ $=\left(2 n f_{\mathrm{OSC}}\right)^{-1}$, where $n$ is the number of inverting stages $(n$ $=7$ ), yielding $34 \mu \mathrm{s}$. This value is comparable with previously published data on $p$-channel ring oscillators. ${ }^{38-40}$

By taking advantage of the ambipolar nature of [70]PCBM transistors, we were also able to fabricate complementarylike inverters comprised of two ambipolar OFETs. Figure 7(a) shows the inverter schematic, while Figs. 7(b) and 7(c) show the transfer characteristics of the inverter operating in the first and the third quadrant, respectively. The transistor dimensions are $L=15 \mu \mathrm{m}, W=20 \mathrm{~mm}$ for "OFET 1 " and $L=20 \mu \mathrm{m}, W=20 \mathrm{~mm}$ for "OFET 2." As can be seen
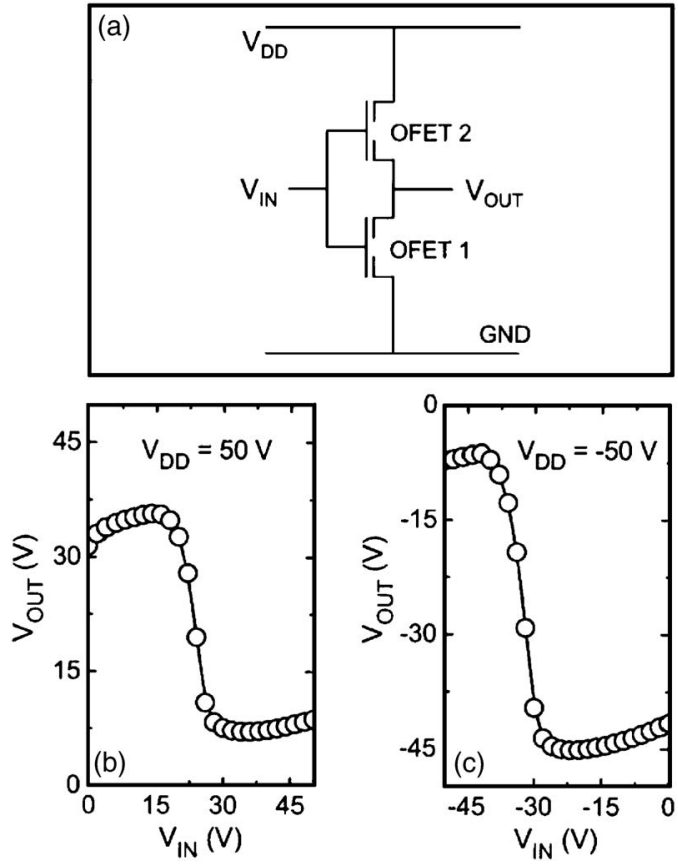

FIG. 7. (a) Schematic circuit of the complementarylike inverter comprised of two [70]PCBM-based ambipolar OFETs. (b) Transfer characteristics of a complementarylike inverter employing transistors with $L=15 \mu \mathrm{m}$ and $W$ $=20 \mathrm{~mm}$.

the inverter exhibits good inversion characteristics in both quadrants with a maximum gain $\left(\right.$ gain $\left.=\partial V_{\mathrm{OUT}} / \partial V_{\mathrm{IN}}\right)$ of approximately 6 . The inefficient pull up and pull down in the voltage transfer curves, observed in both quadrants, are due to the dual operating nature of ambipolar transistors. ${ }^{25,34}$

\section{CONCLUSIONS}

We have shown that organic transistors based on the solution processible methanofullerene [70]PCBM exhibit promising ambipolar charge transport characteristics. To demonstrate the versatility of these transistors we fabricated unipolar ring oscillators and complementarylike inverters. For our fastest $n$-type ring oscillators a signal propagation delay of $34 \mu \mathrm{m} / \mathrm{stage}$ was measured corresponding to an oscillation frequency of $2.1 \mathrm{kHz}$. For the complementarylike voltage inverters a gain of approximately 6 was measured. The advantage of the complementarylike approach demonstrated here over fully complementary logic is the fact that no sequential deposition of a $p$ - and an $n$-channel material is necessary. Instead a single ambipolar semiconductor, which in this particular case can be processed from solution, is required. This approach allows the realization of logic circuits with good noise immunity, a major bottleneck in the today's state-of-the-art unipolar organic circuits.

\section{ACKNOWLEDGMENT}

One of the authors (T.D.A.) gratefully acknowledges financial support from the EC under Project No. HPRN-CT2002-00327 (EUROFET).

${ }^{1}$ G. H. Gelinck, T. C. T. Geuns, and D. M. de Leeuw, Appl. Phys. Lett. 77, 1487 (2000).

${ }^{2}$ D. Voss, Nature (London) 407, 442 (2000). 
${ }^{3}$ H. E. A. Huitema et al., Nature (London) 414, 599 (2001).

${ }^{4}$ C. D. Dimitrakopoulos and P. R. L. Malenfant, Adv. Mater. (Weinheim, Ger.) 14, 99 (2002).

${ }^{5}$ G. H. Gelinck et al., Nat. Mater. 3, 106 (2004).

${ }^{6}$ D. M. de Leeuw, G. H. Gelinck, T. C. T. Geuns, E. van Veenedaal, E. Cantatore, and B. H. Huisman, Tech. Dig. - Int. Electron Devices Meet. 2002, 293.

${ }^{7}$ A. R. Brown, A. Pomp, C. M. Hart, and D. M. de Leeuw, Science 270, $972(1995)$

${ }^{8}$ Z. Bao, A. J. Lovinger, and J. Brown, J. Am. Chem. Soc. 120, 207 (1998)

${ }^{9}$ H. Sirringhaus, N. Tessler, and R. H. Friend, Science 280, 1741 (1998).

${ }^{10}$ A. R. Brown, C. P. Jarrett, D. M. de Leeuw, and M. Matters, Synth. Met. 88, 37 (1997).

${ }^{11}$ B. Crone et al., Nature (London) 403, 521 (2000).

${ }^{12}$ V. C. Sundar, J. Zaumseil, V. Podzorov, E. Menard, R. L. Willet, T. Someya, M. E. Gershenson, and J. A. Rogers, Science 303, 1644 (2004).

${ }^{13}$ H. E. Katz, A. J. Lovinger, J. Johnson, C. Kloc, T. Siegrist, W. Li, Y.-Y. Lin, and A. Dodabalapur, Nature (London) 404, 478 (2000).

${ }^{14}$ R. C. Haddon, A. S. Perel, R. C. Morris, T. T. M. Palstra, A. F. Hebard, and R. M. Fleming, Appl. Phys. Lett. 67, 121 (1995).

${ }^{15}$ R. C. Haddon, J. Am. Chem. Soc. 118, 3041 (1996).

${ }^{16}$ R. Kumashiro et al., Appl. Phys. Lett. 84, 2154 (2004).

${ }^{17}$ T. Kanbara et al., Chem. Phys. Lett. 379, 223 (2003).

${ }^{18}$ S.-I. Kobayashi et al., J. Am. Chem. Soc. 125, 8116 (2003).

${ }^{19}$ K. Shibata, Y. Kubozono, T. Kanbara, T. Hosokawa, A. Fujiwara, Y. Ito, and H. Shinohara, Appl. Phys. Lett. 84, 2572 (2004).

${ }^{20}$ S. Kobayashi, T. Takenobu, S. Mori, A. Fujiwara, and Y. Iwasa, Appl. Phys. Lett. 82, 4581 (2003).

${ }^{21}$ K. Horiuchi, K. Nakada, S. Uchino, S. Hashii, A. Hashimoto, N. Aoki, Y. Ochiai, and M. Shimizu, Appl. Phys. Lett. 81, 1911 (2002).

${ }^{22}$ C. Waldauf, P. Schilinsky, M. Perisutti, J. Hauch, and C. J. Brabec, Adv. Mater. (Weinheim, Ger.) 15, 2084 (2003).

${ }^{23}$ T. D. Anthopoulos, C. Tanase, S. Setayesh, E. J. Meijer, J. C. Hummelen, P. W. M. Blom, and D. M. de Leeuw, Adv. Mater. (Weinheim, Ger.) 16,
2174 (2004).

${ }^{24}$ Th. B. Singh et al., J. Appl. Phys. 97, 083714 (2005).

${ }^{25}$ T. D. Anthopoulos, D. M. de Leeuw, E. Cantatore, S. Setayesh, E. J. Meijer, C. Tanase, J. C. Hummelen, and P. W. M. Blom, Appl. Phys. Lett. 85, 4205 (2004).

${ }^{26}$ J. C. Hummelen, B. W. Knight, F. LePeq, F. Wudl, J. Yao, and C. L. Wilkins, J. Org. Chem. 60, 532 (1995).

${ }^{27}$ M. M. Wienk, J. M. Kroon, W. J. H. Verhees, J. Knol, J. C. Hummelen, P. A. van Hal, and R. A. J. Janssen, Angew. Chem., Int. Ed. 42, 3371 (2003). ${ }^{28}$ J. W. Arbogast and C. S. Foote, J. Am. Chem. Soc. 113, 8886 (1991).

${ }^{29}$ Y. Yang, F. Arias, L. Echegoyen, L. P. F. Chibante, S. Flanagan, A. Robertson, and L. J. Wilson, J. Am. Chem. Soc. 117, 7801 (1995).

${ }^{30}$ A. R. Brown, C. P. Jarret, D. M. de Leeuw, and M. Matters, Synth. Met. 88, 37 (1997).

${ }^{31}$ R. C. Haddon, Acc. Chem. Res. 25, 127 (1992).

${ }^{32}$ M. C. J. M. Vissenberg and M. Matters, Phys. Rev. B 57, 12964 (1998).

${ }^{33}$ H. Sirringhauus, N. Tessler, D. S. Thomas, P. J. Brown, and R. H. Friend, Festkoerperprobleme 39, 101 (1999).

${ }^{34}$ E. J. Meijer et al., Nat. Mater. 2, 678 (2003).

${ }^{35}$ R. J. Chesterfield, C. R. Newman, T. M. Pappenfus, P. C. Ewbank, M. H. Haukaas, K. R. Mann, L. L. Miller, and C. D. Frisbie, Adv. Mater. (Weinheim, Ger.) 15, 1278 (2003).

${ }^{36}$ C. Rost, D. J. Gundlach, S. Karg, and W. Rieb, J. Appl. Phys. 95, 5782 (2004).

${ }^{37}$ E. Cantatore and E. J. Meijer, Proceedings of the 29th European SolidState Circuits Conference, Estoril, Portugal, 16-18 September 2003 (unpublished), p. 29.

${ }^{38}$ Y.-Y. Lin, A. Dodabalapur, R. Sarpeshkar, Z. Bao, W. Li, K. Baldwin, V. R. Raju, and H. E. Katz, Appl. Phys. Lett. 74, 2714 (1999).

${ }^{39}$ W. Fix, A. Ullmann, J. Ficker, and W. Clemens, Appl. Phys. Lett. 81, 1735 (2002)

${ }^{40}$ F. Eder, H. Klauk, M. Halik, U. Zschieschang, G. Schmid, and C. Dehm, Appl. Phys. Lett. 84, 2673 (2004). 